\title{
Preparation and in vitro evaluation of methylene blue films for treatment of oral mucosal diseases
}

\author{
Özgür Esim ${ }^{1}$ \\ (1) University of Health Sciences, Gulhane Faculty of Pharmacy, Department of Pharmaceutical Technology, Ankara, \\ Turkey
}

Date submitted:

Mar 29, 2019

Date accepted:

Apr 11, 2019

Online publication date:

September 15, 2019

\section{Corresponding Author: \\ Özgür Esim \\ University of Health Sciences, Gulhane Faculty of Pharmacy, Department of Pharmaceutical Technology, Ankara, Turkey ozgur.esim@sbu.edu.tr}

Keywords: Methylene Blue, Buccal Film, Pullulan, Maltodextrin, Propylene Glycol.

\begin{abstract}
Aims:The aim of this work was to develop and characterize the buccal films of methylene blue (MB) using natural polysaccharide pullulan and maltodextrin and to evaluate buccal films in vitro for the application in oral mucosal diseases.
\end{abstract}

Methods:The mucoadhesive buccal films of methylene blue were prepared by solvent casting technique using maltodextrin and pullulan at different ratios. Mechanical properties and disintegration time of the films were evaluated and sample sizes were chosen as described in pharmacopeias.

Results:After statistical evaluation using student's t test, it is found that polymer type and concentrations affected both of the disintegration time and mechanical properties $(p<0.05)$. The disintegration time of all the prepared formulations were less than $180 \mathrm{sec}$. and all formulations showed moderate mechanical strength $(0.110 \pm 0.034$ to $0.783 \pm 0.128 \mathrm{kgf})$.

Conclusions:From the experimental data of our study it can be concluded that optimized formulations of MB loaded buccal films can be an adjunct to the therapy to complete eradication of the diseases in future.

\section{Introduction}

Methylene blue $(\mathrm{MB})$ is one of the first ever totally synthetic drugs used in medicine. MB (3, 7-bis (dimethylamino)-phenazathionium chloride tetramethylthionine chloride) is a heterocyclic aromatic compound with the $\mathrm{C}_{16} \mathrm{H}_{18} \mathrm{CIN}_{3} \mathrm{~S}, 3 \mathrm{H}_{2} \mathrm{O}$ molecular formula. It is has a deep blue color and its $10 \mathrm{mg} / \mathrm{mL}$ formulation for intravenous injection. Owing to incompatibility with other strong oxidizing or reducing agents, injectable solutions of $\mathrm{MB}$ contains small amounts of sodium hydroxide or hydrogen chloride to adjust the $\mathrm{pH}$ to $3-4.5$. (1).

MB is commonly used as dye for different type of applications at the outset. One of the most important applications of MB takes place in the treatment of malaria after the observation of the harmful effects of MB on microorganisms (2). Moreover, due to its similarity of reduction potential to oxygen and ability of being reduced by members of the electron transport chain, MB can be used as an antidote in treatment of symptomatic methemoglobinemia (1).

Several clinical uses of MB were also reported in previous studies. For instance, MB can be used as topical agent (at a
$0.1 \%$ solution) in conjunction with polychromatic light to photoinactivate viruses such as herpes simplex (3). MB is also used experimentally to increase vascular tone and myocardial function in patients with septic or anaphylactic shock (4).

Oral lichen planus is a chronic inflammatory oral mucosal disease and Candida albicans is detected in $37 \%$ of oral lichen planus lesions approximately. Candida albicans might play different roles in different mechanisms in two groups of patients suffering from oral premalignant lesions and oral cancers (5). The antibacterial characteristics of MB and the use in the therapy of oral lichen planus was attributed to its photodynamic properties such as its strong absorption at wavelengths longer than $620 \mathrm{~nm}$, where light penetration into tissue is optimal. Because of the well-known low toxicity, photoactive antimicrobial potential and acceptance in the medical field of MB is investigated in order to evaluate the destruction capacity of oral candida albicans and found to be effective (6).

Intraoral drug delivery is an important administration type for local treatment of different oral diseases. The target sites for local drug delivery in the oral cavity include the buccal, sublingual 
and periodontal region. Moreover, these oromucosal regions can be used for the treatment of systemic diseases as drug molecules are directly transferred into systemic circulation. The classical intraoral formulation has been the lozenge. However, during the past decade, tablets, gels and films were also studied (7).

In local treatment of oral mucosal diseases, application of drugs as solutions or oromucosal drug delivery systems takes part generally (7). Considering the MB is a dye, application of this drug as solution results in coloration of mouth and other application pathways. Hence, other types of drug delivery systems containing MB such as buccal films must be preferred to treat the local diseases in oral mucosa.

Buccal films are novel drug delivery systems which can release drug in the desired duration of action (8). Different polymers are used to formulate the buccal films and these polymers adjust the drug release and bioadhesion for mucosal attachment. Flexible films/patches have been prepared either by solvent casting or hot melt extrusion technique to deliver drugs directly to a mucosal membrane (9).
The aim of this work was to develop and characterize the buccal film of MB using natural polysaccharide pullulan and maltodextrin and to evaluate films for its buccal delivery for the local treatment of oral mucosal diseases. The mucoadhesive buccal films of MB were prepared by solvent casting technique.

\section{Methods}

MB (Sigma, USA), Maltodextrin (dextrose equivalent 13.017.0) (Aldrich, USA), and Pullulan (Xi'an Sonwu Biotech, China) were used. All other chemicals were of analytical grade.

\section{Preparation of MB Buccal Films}

In preformulation studies, two film-forming polymers were used, namely, maltodextrin and pullulan. Propylene Glycol (PG), Polyethylene Glycol 400 (PEG 400) and Glycerol were used as plasticizer. The films were prepared by solvent-casting technique. The film-forming materials and MB were mixed in distilled water for $2 \mathrm{~h}$ at $2000 \mathrm{rpm}$ with a mechanic stirrer (Heidolph, Germany). The plasticizer was added to this solution and stirred for another $1 \mathrm{~h}$. The dispersion was used after at least $24 \mathrm{~h}$ of rest to remove all the entrapped air bubbles. The dispersion was cast onto glass petri dishes and then kept for dry-

\begin{tabular}{|lcccccc}
\hline \multicolumn{7}{l}{ Table 1. Prepared film formulations and their characteristics } \\
\hline & $\begin{array}{c}\text { Maltodextrin } \\
\text { Ratio }\end{array}$ (\%) & $\begin{array}{c}\text { Pullulan } \\
\text { Ratio (\%) }\end{array}$ & $\begin{array}{c}\text { Propylene Glycol } \\
\text { Ratio }(\%)\end{array}$ & $\begin{array}{c}\text { Methylene } \\
\text { Blue (\%) }\end{array}$ & $\begin{array}{c}\text { Disintegration } \\
\text { (sec) }\end{array}$ & $\begin{array}{c}\text { Mechanical Strength } \\
\text { (kgf) }\end{array}$ \\
\hline F1 & 3 & 1 & 10 & 1 & $26 \pm 6$ & $0.617 \pm 0.041$ \\
\hline F2 & 3 & 2 & 10 & 1 & $35 \pm 7$ & $0.683 \pm 0.074$ \\
\hline F3 & 1 & 1 & 10 & 1 & $9 \pm 2$ & $0.130 \pm 0.019$ \\
\hline F4 & 2 & 2 & 10 & 1 & $30 \pm 4$ & $0.594 \pm 0.108$ \\
\hline F5 & 2 & 3 & 10 & 1 & $43 \pm 11$ & $0.396 \pm 0.049$ \\
\hline F6 & 2 & 1 & 10 & 1 & $20 \pm 2$ & $0.371 \pm 0.089$ \\
\hline F7 & 3 & 3 & 10 & 1 & $47 \pm 4$ & $0.749 \pm 0.071$ \\
\hline F8 & 1 & 3 & 10 & 1 & $16 \pm 4$ & $0.130 \pm 0.058$ \\
\hline F9 & 1 & 2 & 10 & 1 & $8 \pm 2$ & $0.176 \pm 0.033$ \\
\hline F10 & 1 & 3 & 15 & 1 & $31 \pm 5$ & $0.157 \pm 0.057$ \\
\hline F11 & 2 & 1 & 15 & 1 & $46 \pm 15$ & $0.186 \pm 0.061$ \\
\hline F12 & 3 & 1 & 15 & 1 & $42 \pm 2$ & $0.486 \pm 0.092$ \\
\hline F13 & 1 & 1 & 15 & 1 & $18 \pm 1$ & $0.160 \pm 0.046$ \\
\hline F14 & 3 & 3 & 15 & 1 & $27 \pm 3$ & $0.419 \pm 0.104$ \\
\hline F15 & 2 & 2 & 15 & 1 & $180 \pm 83$ & $0.293 \pm 0.059$ \\
\hline F16 & 3 & 2 & 15 & 1 & $83 \pm 4$ & $0.388 \pm 0.079$ \\
\hline F17 & 2 & 3 & 15 & 1 & $34 \pm 6$ & $0.267 \pm 0.087$ \\
\hline F18 & 1 & 2 & 15 & 1 & $27 \pm 5$ & $0.149 \pm 0.040$ \\
\hline F19 & 2 & 1 & 5 & 1 & $33 \pm 8$ & $0.783 \pm 0.128$ \\
\hline F20 & 1 & 2 & 5 & 1 & $8 \pm 2$ & $0.191 \pm 0.027$ \\
\hline F21 & 3 & 3 & 5 & 1 & $9 \pm 3$ & $0.611 \pm 0.073$ \\
\hline F22 & 2 & 3 & 5 & 1 & $16 \pm 2$ & $0.780 \pm 0.081$ \\
\hline F23 & 1 & 1 & 5 & 1 & $8 \pm 3$ & $0.110 \pm 0.034$ \\
\hline F24 & 3 & 2 & 5 & 1 & $12 \pm 4$ & $0.639 \pm 0.108$ \\
\hline F25 & 3 & 1 & 5 & 1 & $11 \pm 3$ & $0.362 \pm 0.059$ \\
\hline F26 & 2 & 2 & 5 & 1 & $29 \pm 6$ & $0.627 \pm 0.084$ \\
\hline F27 & 1 & 3 & 5 & $9 \pm 2$ & $0.158 \pm 0.047$ \\
\hline & & & & 1 & & \\
\hline
\end{tabular}


ing in vacuum oven (Heidolph, Germany) at $60^{\circ} \mathrm{C}$ for $2 \mathrm{~h}$. The resultant film was cut into the dimensions including the desired amount of MB. Films having cuts, air bubbles, or imperfections were excluded from the study.

Following the ascertainment of the suitable polymer and plasticizer type and concentrations, $1 \%, 2 \%$ and $3 \%$ concentrations of pullulan and maltodextrin and $5 \%, 10 \%$ and $15 \%$ concentrations of propylene glycol were used and analyzed in order to determine all the effects of the substances within the film structure (Table 1).

\section{Characterization of MB Films}

\section{In vitro Disintegration Time}

The test was performed using the USP disintegration testing apparatus (Sotax, Switzerland). The film size required for dose delivery $(2 \times 2 \mathrm{~cm})$ was placed in the tubes. Test was performed using distilled water as disintegration media. The time required for the film disintegration was noted as in vitro disintegration time. Six replicates were done in accordance with the pharmacopeia (10).

\section{Measurement of Mechanical Strength}

Mechanical strength of the films were evaluated using a Universal Testing Instrument with a $10 \mathrm{~N}$ load cell (Tinius Olsen H5KS, USA). Film strip in $2 \mathrm{~cm}^{2}$, free from air bubbles or physical imperfections, were held between two clamps. During measurement, the strips were pulled by the top clamp at a rate of $1.0 \mathrm{~mm} / \mathrm{s}$. The force was measured when the films broke. Results of unbroken film samples were excluded in calculations. Measurements were run in six replicates for each film (11).

\section{Application Stability of Films}

Stability of buccal films in biological fluids is an important factor for determination of both suitability of formulation for application and possible side effects in buccal mucosa. Films were evaluated in terms of the stability in artificial saliva. For this purpose, films were wetted using artificial saliva and physical imperfections were recorded $(n=6)$.

\section{Statistical analysis}

Data are expressed as the mean \pm SEM. Statistical analysis was performed using Student's t-test with GraphPad prism software. Populations were selected in accordance with pharmacopeia and standard methods $(10,12)$. The level of probability at 0.05 was used as the level of significance.

\section{Results}

\section{Preformulation Studies}

In the preformulation studies; distilled water was used for the formation buccal films considering lack of toxicity. At first, pulIulan $(2 \%)$ and maltodextrin $(2 \%)$ were dissolved in $16 \mathrm{~mL}$ of water and $4 \mathrm{~mL}$ of PEG 400 and poured into molds. Molds were left at room temperature for 12 hours to remove air bubbles. After obtaining clear solution, the prepared formulation was allowed to dry at $60{ }^{\circ} \mathrm{C}$ in the oven. At each hour, drying samples were evaluated for forming of the appropriate film and optimum time of drying was obtained as $2 \mathrm{~h}$. Acquired films of mentioned formulation was found opaque and brittle which is not acceptable for buccal application. With the purpose of determining the reason of this interaction, $500 \mathrm{mg}$ of pullulan or the same amount of maltodextrin was dissolved separately in $9 \mathrm{ml}$ of distilled water and $1 \mathrm{~mL}$ of PEG 400 was added to both solutions.
Pullulan and PEG 400 were formed an opaque and brittle film after evaporation. Owing to the obtained results, PEG 400 was withdrawn from the study and it is concluded other plasticizers for the preparation of pullulan films may be appropriate.

From this point of view, glycerol and propylene glycol were investigated as plasticizer in pullulan-maltodextrin films. Pullulan $(2 \%)$ and maltodextrin $(2 \%)$ was dissolved in $9 \mathrm{~mL}$ of water and $1 \mathrm{~mL}$ of both plasticizers were added separately and kept at 60 ${ }^{\circ} \mathrm{C}$ for 2 hours. When glycerol was used as plasticizer, transparent but sticky film was formed which was not also acceptable. However, the utilization of propylene glycol as plasticizer resulted in films having adequate properties. As a consequence, propylene glycol was chosen as plasticizer.

\section{Characterization of MB Films}

Totally 27 film formulations were prepared and their disintegration and mechanical strengths were evaluated using compendia methods (Table 1) $(10,12,13)$.

\section{Disintegration of Films}

One of the most important quality control parameters for oromucosal drug delivery systems is disintegration time. There is no formal disintegration test method for buccal films. But according to United States Pharmacopoeia, the buccal tablets must disintegrate in $4 \mathrm{~h}$. Hence, United States Pharmacopoeia Disintegration Test for buccal tablets is used as reference (10). The experiment was carried out in distilled water using the disintegration tester (Sotax, Switzerland) $(2,3)$. It was very difficult to observe the disintegration of active substance-free films not allowing the clearly record of disintegration times. But, MB also solved this problem due to its color (Figure 1).

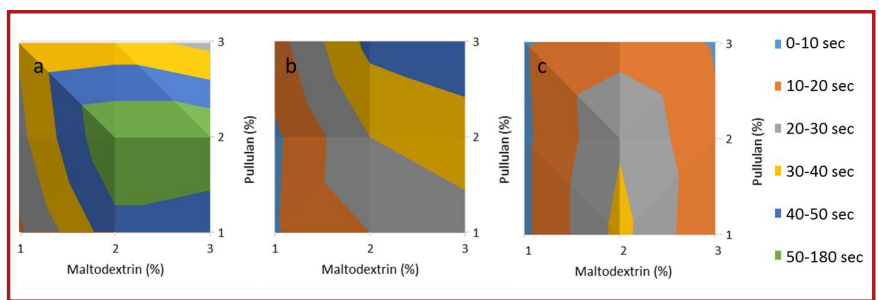

Figure 1. Effect of polymer type and concentration on disintegration time $(15 \%$ PG (a), $10 \%$ PG (b) ve $5 \%$ PG (c). $(n=6)$

As a result of the examination of prepared films, it was found that all the prepared film formulations meet the requirements in pharmacopeia described as "all the formulations must disintegrate within 4 h" (Figure 2) (10). Higher concentration of each polymer caused retardation in the disintegration time. For instance, when the pullulan concentration increased from $1 \%$ to $3 \%$ in films with $3 \%$ maltodextrin and $10 \%$ propylene glycol, the disintegration time of films were recorded as $47 \pm 4 \mathrm{sec}$. which was $26 \pm 6 \mathrm{sec}$. $(p<0.05)$ (Table 1$)$. Moreover, the increase in the maltodextrin concentration from $1 \%$ to $3 \%$ in films with $3 \%$ pullulan and $10 \%$ propylene glycol the disintegration time changed from $16 \pm 4 \mathrm{sec}$. to $47 \pm 4 \mathrm{sec}$. Increase in the

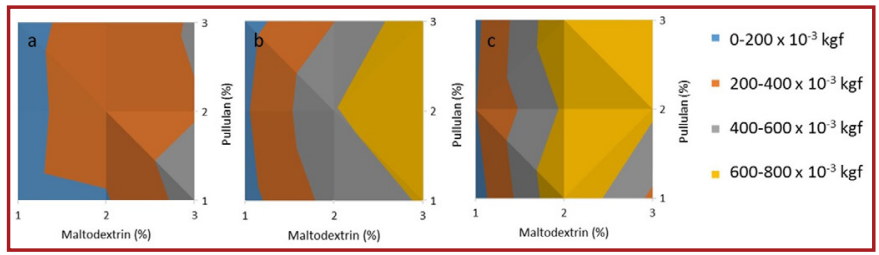

Figure 2. Effect of polymer type and concentration on mechanical properties of films (15\% PG (a), 10\% PG (b) ve 5\% PG (c). ( $n=3)$ 
total concentration of maltodextrin and pullulan also increased the disintegration time $(p<0.05)$.

In the presence of the same total concentration of polymers; higher pullulan ratios had shorter disintegration time than the same concentration of maltodextrin $(26 \pm 6 \mathrm{sec}$. for $\mathrm{F} 1$ and 16 \pm 4 sec. for $F 8)$, which may have relation with its lower viscosity (14). Disintegration time was also found to be increased with the increase in the amount of plasticizer used $(p<0.05)$. This coincides with our previous study results which also showed that as the concentration of propylene glycol increases the disintegration time increases (15).

As an important parameter to show the durability of the films, mechanical strength of the films were evaluated. Increase in the pullulan concentration resulted in the higher mechanical strength $(0.617 \pm 0.041 \mathrm{kgf}$ for $\mathrm{F} 1$ and $0.749 \pm 0.071 \mathrm{kgf}$ for F7) $(p<0.05)$ (Table 1). Maltodextrin and propylene glycol concentration is also affected the mechanical strength in the same manner but in low pullulan concentrations the effect of maltodextrin was not significantly different $(p>0.05)(0.130 \pm 0.019$ kgf for F3 and $0.130 \pm 0.058 \mathrm{kgf}$ for F8) (Figure 2). Unfortunately, the test method for determining mechanical strength was not suitable for all film types such as sticky and brittle films prepared using PEG 400 or glycerol. Since the lack of a standard method or threshold for sufficient mechanical strength of thin film preparations for oral use, the aforementioned test could not be applied to all film formulations (16).

\section{Application Stability of Films}

After application of artificial saliva on buccal films, films were observed for the possible physical changes. However no physical imperfections were recorded which shows that buccal films are stable in artificial saliva.

\section{Discussion}

By the determination of the appropriate polymers in preformulation studies, the effect of polymer type and concentrations were investigated. In vitro disintegration time of all the prepared films showed a disintegration time less than 3 minutes. The results were comparable with the previous studies (17). It is found that higher polymer ratios significantly increase the disintegration time $(p<0.05)$. This situation may be based upon low polymer concentrations causing increased surface wetting and water penetration which resulted in faster disintegration. It is in accordance to Li et al. who also found that the disintegration time is significantly decreased by decreasing the molecular weight of the film forming polymer (18).

Among different pullulan-maltodextrin ratios used, the higher total polymer ratios increased the disintegration time. The reason that maltodextrin and pullulan are oligosaccharides it increases the erosion of the polymer causing rapid disintegration. The higher fraction of these polymers which has viscosity facilitates lower penetration of water into the film structure leading to increased disintegration time (17).

Mechanical strength results showed that all the films have moderate strength values and increasing polymer concentration is a factor for the increase in strength of films. It was observed that combination of polymers lead to the increase in the strength and formation of more elastic. This may be attributed to increased moisture content of pullulan and maltodextrin films which could exert increased plasticizing effects on polymeric glass structure in compliance with the results obtained by Sallam et al. who prepared salbutamol sulphate films and observed that the increase in pullulan and maltodextrin ratios brought about an increase in the mechanical strength (17). Also, Khanzadi et al. found that the mixture of some sort of polymers and pullulan possess improved mechanical strength compared to the pure pullulan film (19).

\section{Conclusion}

According to the results, the use pullulan and maltodextrin at a rate of $1-3 \%$ to formulate MB films is appropriate. From the experimental data of our study, it can be concluded that optimized formulations of MB loaded buccal films can be an adjunct to the therapy to complete eradication of the diseases in future.

Acknowledgement: The study was designed by OE, measurements were made by $\mathrm{OE}$, the data was collected and analyzed by OE, manuscript was written by OE. This study received no financial support.

Conflict of Interest: The author declared he does not have anything to disclose regarding with the conflict of interest with respect to this manuscript.

\section{References}

1. Lo JC, Darracq MA, Clark RF. A review of methylene blue treatment for cardiovascular collapse. J Emerg Med. 2014;46(5):670-679.

2. Krafts K, Hempelmann E, Skórska-Stania A. From methylene blue to chloroquine: a brief review of the development of an antimalarial therapy. Parasitol Res. 2012;111(1):1-6.

3. Felber TD, Smith EB, Knox JM, Wallis C, Melnick JL. Photodynamic inactivation of herpes simplex: report of a clinical trial. JAMA. 1973;223(3):289-292.

4. Clifton JI, Leikin JB. Methylene blue. Am J Ther. 2003;10(4):289-291.

5. Mehdipour M, Taghavi Zenouz A, Hekmatfar S, Adibpour M, Bahramian A, Khorshidi R. Prevalence of Candida species in erosive oral lichen planus. J Dent Res Dent Clin Dent Prospects. 2010;4(1):14-16.

6. Teichert M, Jones J, Usacheva M, Biel M. Treatment of oral candidiasis with methylene blue-mediated photodynamic therapy in an immunodeficient murine model. Oral Surg Oral Med Oral Pathol Oral Radiol Endod. 2002;93(2):155-160.

7. Brandl M, Bauer-Brandl A. Oromucosal drug delivery: Trends in in-vitro biopharmaceutical assessment of new chemical entities and formulations. Eur J Pharm Sci. 2019;128:112-117.

8. Reddy PC, Chaitanya K, Madhusudan Rao Y. A review on bioadhesive buccal drug delivery systems: current status of formulation and evaluation methods. Daru. 2011;19(6):385-403.

9. Morales JO, McConville JT. Manufacture and characterization of mucoadhesive buccal films. Eur J Pharm Biopharm. 2011;77(2):187-199.

10. The United States Pharmacopeia, USP 30/The National Formulary, NF 25. Rockville, MD: US Pharmacopeial Convention. Inc; 2007. 
11. Mashru R, Sutariya V, Sankalia M, Parikh P. Development and evaluation of fast-dissolving film of salbutamol sulphate. Drug Dev Ind Pharm. 2005;31(1):25-34.

12. Cilurzo F, Cupone IE, Minghetti P, Selmin F, Montanari L. Fast dissolving films made of maltodextrins. Eur J Pharm Biopharm. 2008;70(3):895-900.

13. European pharmacopoeia. Strasbourg, France: European Directorate for the Quality of Medicines, Council of Europe; 2008.

14. Matsumura $Y$, Satake C, Egami M, Mori T. Interaction of gum arabic, maltodextrin and pullulan with lipids in emulsions. Biosci Biotechnol Biochem. 2000;64(9):18271835.

15. Esim O, K. Ozkan C, Kurbanoglu S, et al. Development and in vitro/in vivo evaluation of dihydroergotamine mesylate loaded maltodextrin-pullulan sublingual films. [published online Feb 4, 2019]. Drug Dev Ind Pharm. doi: 10.1080/03639045.2019.1578788.

16. Preis M, Woertz C, Schneider K, et al. Design and evaluation of bilayered buccal film preparations for local administration of lidocaine hydrochloride. Eur J Pharm Biopharm. 2014;86(3):552-561.

17. Mohamed Sallam N, Abdel-Basset Sanad R, Abdelfadil Zeneldin M. Development of salbutamol sulphate sublingual films in pullulan matrix for enhanced bioavailability \& clinical efficacy. Curr Drug Deliv. 2017;14(4):503-515.

18. Li X-Q, Ye Z-M, Wang J-B, et al. Mucoadhesive buccal films of tramadol for effective pain management. Rev Bras Anestesiol. 2017;67(3):231-237.

19. Khanzadi M, Jafari SM, Mirzaei H, Chegini FK, Maghsoudlou Y, Dehnad D. Physical and mechanical properties in biodegradable films of whey protein concentratepullulan by application of beeswax. Carbohydr Polym. 2015;118:24-29. 